It was found to be acceptable by the community. It is also expected to be feasible and sustainable as very low cost and locally available technology and resources were utilised. Large scale piloting is necessary to explore its effectiveness and ability to scale up all over the country.

\section{EXPERIENCE FROM COMMUNITY BASED CHILDHOOD BURN PREVENTION PROGRAMME IN BANGLADESH: IMPLICATION FOR LOW RESOURCE SETTING}

doi:10.1136/injuryprev-2012-040580c.8

${ }^{1,2}$ SR Mashreky*, ${ }^{1} \mathrm{~A}$ Rahman, ${ }^{2} \mathrm{~L}$ SvanstrÖm, ${ }^{3} \mathrm{MJ}$ Linnan, ${ }^{4} \mathrm{~S}$ Shafinaz, ${ }^{1,2} \mathrm{~F}$ Rahman. ${ }^{1}$ Centre for Injury Prevention and Research, Bangladesh (CIPRB), Bangladesh;

${ }^{2}$ Department of Public Health Science, Karolinska Institute, Sweden; ${ }^{3}$ The Alliance for Safe Children, Thailand; ${ }^{4}$ UNICEF, Bangladesh

A comprehensive community-based burn prevention framework was developed for rural Bangladesh taking into consideration the magnitude, consequences of burns, risk factors of childhood burn, health seeking behaviour of parents after a burn injury of a child and the perception of community people. This paper explains the comprehensive framework of the childhood burn prevention programme and describes its acceptability, feasibility and sustainability.

A number of methodologies were adopted in developing the framework, such as, (i) building up relevant information on childhood burn and prevention methods, (ii) arranging workshops and consultation meetings with experts and related stakeholders and (iii) piloting components of the framework on a small scale.

Lack of supervision of the children, hazardous environment at home and the low level awareness about childhood burn and other injuries were identified as the major attributes of childhood burn in Bangladesh. To address these factors 'Triple S' strategies were identified for the prevention framework.

These strategies are:

- Safe environment.

- Supervision.

- Skill development.

According to these strategies, home safety, community crèche, school safety, formation of community groups and general awareness activities were identified as the different components of the childhood burn prevention framework in rural Bangladesh. The framework was piloted in a small scale to explore its feasibility acceptability and sustainability. 